\title{
Über die Notwendigkeit der wirkungsorientierten Analytik in einer umfassenden Wasserforschung
}

\author{
Henner Hollert • Evelyn Claus • Wolfgang Ahlf • Werner Brack • Thomas Braunbeck • \\ Sebastian Hudjetz • Werner Manz • Hans-Toni Ratte • Georg Reifferscheid • Andreas Schäffer • \\ Tobias Schulze $\cdot$ Jan Schwarzbauer $\cdot$ Peter Heininger
}

Eingegangen: 28. April 2009 / Akzeptiert: 2. Mai 2009/Online veröffentlicht: 20. Mai 2009

(C) Springer-Verlag 2009

\begin{abstract}
Wasser ist nicht nur der Ursprung allen Lebens auf der Erde, sondern mit einem geschätzten Volumen von 1,4 Mrd. Kubikkilometern auch sein größter und wichtigster Rohstoff. Die Agenda 21 (1992) der Vereinten Nationen beschreibt für alle wesentlichen Politikbereiche die Anforderungen an eine umweltgerechte und nachhaltige Entwicklung. Zwei Kapitel dieser Agenda sind dem Thema Wasser - dem Schutz der Meere und Küstengewässer sowie dem Schutz der Süßwasserressourcen - gewidmet. Steigender Wasserverbrauch, nicht-nachhaltige Nutzungsformen der Wasserressourcen und zunehmende Wasserverschmutzung führen in vielen Regionen der Welt zu einer bedrohlichen Verknappung des Trinkwassers und gefährden den Erhalt von Naturräumen und Ökosystemen. Wasser ist deshalb eines der zentralen Themen des globalen Wandels, zu dessen Bewältigung auch die Wasserforschung substanziell beitragen muss. In dieser
\end{abstract}

Verantwortliche Herausgeber: Jan Schwarzbauer · Peter Heininger · Evelyn Claus

Henner Hollert $(\square) \cdot$ Sebastian Hudjetz

Lehr- \& Forschungsgebiet Ökosystemanalyse,

Institut für Umweltforschung (Biologie V),

Fakultät für Mathematik, Informatik und Naturwissenschaften, RWTH University Aachen,

Worringerweg 1, 52074 Aachen, Deutschland

E-Mail: Henner.Hollert@bio5.rwth-aachen.de

Evelyn Claus · Werner Manz · Georg Reifferscheid · Peter Heininger Bundesanstalt für Gewässerkunde (BfG),

Abteilung G Qualitative Gewässerkunde,

Am Mainzer Tor 1, 56068 Koblenz, Deutschland

E-Mail: claus@bafg.de, heininger@bafg.de

Jan Schwarzbauer

Lehrstuhl für Geologie, Geochemie und Lagerstätten des Erdöls und der Kohle (LEK), RWTH Aachen University,

Lochnerstraße 4-20, 52056 Aachen, Deutschland

E-Mail: schwarzbauer@1ek.rwth-aachen.de
UWSF-Ausgabe beginnt eine Serie Wasser - elementare und strategische Ressource des 21. Jahrhunderts von Lehn und Parodi, mit der in drei Beiträgen die Hintergründe einer konstatierten ,globalen Wasserkrise“ aufgearbeitet und Vorschläge eines nachhaltigen Umgangs mit Wasserressourcen skizziert werden (Lehn und Parodi 2009).

Die anhaltende Belastung aquatischer Ökosysteme mit vom Menschen hergestellten Fremdstoffen (Xenobiotika) bleibt trotz der unbestreitbaren großen Fortschritte in der Gewässerreinhaltung auch unter den Bedingungen der modernen westlichen Gesellschaften eine der größten Herausforderungen. Die Gründe dafür sind vielschichtig. Abwässer aus diversen menschlichen Aktivitäten tragen jährlich weltweit etwa 600 Mio.t Schadstoffe in Gewässer ein; von über 100.000 registrierten chemischen Verbindungen befinden sich zwischen 30.000 und 70.000 in nennenswerten Mengen in täglichem Gebrauch (Schwarzenbach et al. 2006). Die Identifikation von Umweltschadstoffen, die Auf-

Wolfgang Ahlf

Institut für Umwelttechnik und Energiewirtschaft, TUHH,

Eißendorfer Straße 40, 21073 Hamburg, Deutschland

Thomas Braunbeck

Universität Heidelberg, Institut für Zoologie,

Im Neuenheimer Feld 230, 69120 Heidelberg, Deutschland

Werner Brack · Tobias Schulze

Helmholtz Zentrum für Umweltforschung - UFZ,

Department für Wirkungsorientierte Analytik,

Permoserstraße 15, 04318 Leipzig, Deutschland

Andreas Schäffer · Hans-Toni Ratte

Lehrstuhl für Umweltbiologie \& Chemodynamik,

Institut für Umweltforschung (Biologie V),

Fakultät für Mathematik, Informatik und Naturwissenschaften,

RWTH University Aachen,

Worringerweg 1, 52074 Aachen, Deutschland 
klärung der Stoffströme in der Umwelt und deren Wirkung auf Mensch und Ökosystem stellen uns vor ein enormes Problem, dessen Komplexität erst in Anfängen verstanden ist. Für viele Chemikalien gibt es keine umweltrelevanten Daten, und wir wissen in den meisten Fällen nicht, wie diese Substanzen die körperliche und geistige Entwicklung des Menschen sowie die Umwelt beeinflussen können. Mit der Europäischen Chemikalienrichtlinie REACh (EC 2007) wird ein Weg aufgezeigt, diese Situation schrittweise zu verbessern. Im Hinblick auf die Vielfalt von synthetischen chemischen Verbindungen stellen mögliche Synergieeffekte und Transformationsphänomene in der Umwelt oder im Körper eine weitere Ebene der Komplexität dar, die wir bestenfalls ansatzweise verstehen. Nachdem sich die Toxikologie und Ökotoxikologie in der Vergangenheit vor allem mit der akut toxischen Wirkung definierter prioritärer Stoffe auf Organismen befassten, konnten deren Konzentrationen in der Umwelt durch Maßnahmen des Gewässerschutzes zunächst deutlich reduziert werden. So haben sich die Quecksilberemissionen in die Oberflächengewässer Deutschlands zwischen 1985 und 2000 um 85\% verringert (Bundesministerium für Umwelt, Naturschutz und Reaktorsicherheit 2006), oder - wie im Beitrag von Rüdel et al. (2009) in dieser Ausgabe dargestellt - die Konzentrationen von Organozinnverbindungen in biologischen Proben aus Nord- und Ostsee. Da aber z. B. trotz der Verbesserung der Wasserqualität die Abnahme der Artenvielfalt aquatischer Ökosysteme und auch Bestandsrückgänge von Fischen (Braunbeck et al. 2009; Keiter et al. 2009) nicht gestoppt werden konnte, wendet sich das Augenmerk der ökotoxikologischen Forschung zunehmend einer komplexen Betrachtung der gesamten Schadstoffsituation eines Gewässers zu (vgl. z.B. Brack et al. 2005a,b, 2007, 2009; Reifferscheid et al. 2005: Claus et al. 2009a; Hollert et al. 2007, 2009a,b). Viele Stoffe der in einem Gewässer vorkommenden komplexen Schadstoffmischungen treten nur in geringen Spuren auf und sind deshalb analytisch vor dem Hintergrund einer interferierenden Matrix kaum zu fassen. Sie entfalten jedoch für sich oder im Gemisch mit anderen Komponenten erhebliche Schadwirkungen gegenüber aquatischen Organismen und ggf. über eine Anreicherung in der Nahrungskette auch gegen Menschen.

Vor dem beschriebenen Hintergrund sollten akute und mechanismusspezifische Biotestverfahren stärker für eine Identifizierung eines vermeintlichen Schädigungspotenzials eingesetzt werden, um den Nachteil auszugleichen, den ein rein chemisches Monitoring in sich birgt (vgl. Hollert et al. 2009a; Reifferscheid et al. 2009). Es zeigt sich derzeit in mehreren laufenden nationalen und internationalen Forschungsprojekten, dass der Ansatz der wirkungsorientierten Analytik hervorragend geeignet ist, bisher unbekannte bzw. durch Monitoringprogramme nicht erfasste Schadstoffe mit adverser Wirkung zu identifizieren (z.B. Brack et al. 2005a,b, 2007, 2009; Hollert et al. 2007, 2009a,b; Reifferscheid et al. 2005, 2009). In dieser Ausgabe soll daher in einer Beitragsreihe ein Überblick gegeben werden über moderne Ansätze und Fallstudien zur wirkungsorientierten Analytik in der aquatischen Umwelt. Die insgesamt acht Kurzbeiträge wurden anlässlich eines gemeinsamen Workshops der RWTH Aachen und der Bundesanstalt für Gewässerkunde erarbeitet (Claus et al. 2009b).

Bereits jetzt lässt sich aus dem erwiesenen ökotoxikologischen Potenzial und den resultierenden ökologischen Wirkungen schlussfolgern, dass nicht-prioritäre Schadstoffe bei der weiteren Umsetzung der EG-WRRL stärkerer Aufmerksamkeit bedürfen, wenn zentrale Ziele nicht verfehlt werden sollen (vgl. Hollert et al. 2007). Entsprechender Handlungsbedarf ergibt sich auch im Zuge prognostizierter Veränderungen in den Gewässereigenschaften, der Landnutzung und Wasserwirtschaft als Folge des globalen und des Klimawandels. Laut der deutschen Anpassungsstrategie an den Klimawandel (DAS), die vom Bundeskabinett am 17. Dezember 2008 beschlossen wurde, wird der Bund die Umsetzung der Wasserrahmenrichtlinie und der Hochwasserrisikomanagementrichtlinie durch Forschungsaktivitäten zu methodischen Aspekten unterstützen. Insbesondere die Kombination aus wirkungsorientierter Analytik (Kombination aus Fraktionierungsmethoden, biologischen Wirktests und nachfolgenden chemischen Analysen der toxischen Teilproben) und Weight-of-Evidence-Strategien ${ }^{1}$ kann in Zukunft zielorientiert für die Identifizierung von Schadstoffen sowie ihrer adversen Wirkungen und Quellen genutzt werden und so Hinweise für eine effiziente Ausrichtung und Fortschreibung der Bewirtschaftungspläne nach WRRL liefern. Über Weight-of-Evidence-Ansätze kann eine Überprüfung der Relevanz der Laborbefunde für die Situation im Freiland gelingen, und mittels effektdirigierter Analysen die Identifizierung der bisher unbekannten Schadstoffe mit hoher biologischer Wirksamkeit erfolgen (z. B. Brack et al. 2005a; Hollert et al. 2009b).

Um Fehlinterpretationen und falsche Prioritätensetzung zu vermeiden, sollte bei der wirkungsorientierten Analytik neben den eigentlichen Effekten auch die Bioverfügbarkeit berücksichtigt werden. Hier können einerseits Sedimentkontakttests eingesetzt werden, die a priori die Bioverfügbarkeit von sedimentgebundenen Schadstoffen mit abbilden (Ahlf et al. 2009; Feiler et al. 2005, 2009). Andererseits können milde Extraktionsverfahren (z.B. mit TENAX) und vertei-

\footnotetext{
${ }^{1}$ Weight-of-Evidence-Studien versuchen über eine holistische Betrachtungsweise mit verschiedenen Beweislinien (Lines-of-Evidence) den ökologischen Systemzustand zu erfassen. Insbesondere die gemeinsame Analyse aller drei Komponenten aus dem Triade-Konzept, (a) Konzentration und Verbleib der Chemikalien, (b) biologische Effekte in (labornahen) bioanalytischen Methoden und (c) ökologische Relevanz sind als Beweislinien für eine aussagekräftige Ökosystembewertung sehr zielführend.
} 
lungsbasierte Dosierung helfen, die Bioverfügbarkeit in der wirkungsorientierten Analytik zu berücksichtigen (Brack et al. 2009).

Obwohl in der wirkungsorientierten Analytik im deutschsprachigen Raum in den vergangenen Jahren große Erfolge erzielt werden konnten, wird dieser Bereich derzeit - wie die gesamte Wasserforschung - nur sehr unzureichend von den großen nationalen Förderinstitutionen wie BMBF, DFG, UBA und DBU unterstützt. Um nicht den Anschluss an die internationale Wasserforschung zu verlieren, und auch, um im Sinne der High-Tech-Strategie des BMBF und der German Waters Initiative konkurrenzfähig zu bleiben, müssen die Förderaktivitäten im Bereich der Wasserforschung unbedingt gestärkt werden. Eine verstärkte Förderung der wirkungsorientierten Analytik wäre auch ein aktiver Beitrag der von Schaeffer et al. (2009) geforderten dringend notwendigen Stärkung der Umweltchemie und Ökotoxikologie.

\section{Literatur}

Agenda 21 der UN (1992) http://www.un.org/Depts/german/conf/ agenda21/agenda_21.pdf

Bundesministerium für Umwelt, Naturschutz und Reaktorsicherheit (2006) Umweltpolitik. Wasserwirtschaft in Deutschland. Teil 2 Gewässergüte. Bundesministerium für Umwelt, Naturschutz und Reaktorsicherheit, Berlin, S 10f

Ahlf W, Matthäi A, Offermann K (2009) Bedeutung der Expositionswege auf Bioakkumulation und Wirkung in C. elegans. Beitragsreihe Wirkungsorientierte Analytik in der Aquatischen Umwelt. Umweltwiss Schadst Forsch 21(3):245-247

Brack W, Bakker J, de Deckere E, Deerenberg C, van Gils J, Hein M, Jurajda P, Kooijman B, Lamoree M, Lek S, Lopez de Alda MJ, Marcomini A, Munoz I, Rattei S, Segner H, Thomas K, von der Ohe PC, Westrich B, de Zwart D, Schmitt-Jansen M (2005a) MODELKEY. Models for assessing and forecasting the impact of environmental key pollutants on freshwater and marine ecosystems and biodiversity. Environ Sci Pollut Res 12:252-256

Brack W, Bandow N, Schwab K, Schulze T, Streck G (2009) Identifizierung toxischer Verbindungen in Sedimenten: Ansätze zur Integration von Wirkung und Bioverfügbarkeit. Beitragsreihe Wirkungsorientierte Analytik in der Aquatischen Umwelt. Umweltwiss Schadst Forsch 21(3):240-244

Brack W, Klamer HJ, Lopez de Alda M, Barcelo D (2007) Effectdirected analysis of key toxicants in European river basins. a review. Environ Sci Pollut Res 14:30-38

Brack W, Schirmer K, Erdinger L, Hollert H (2005b) Effect-directed analysis of mutagens and ethoxyresorufin-O-deethylase inducers in aquatic sediments. Environ Toxicol Chem 24:2445-2458

Braunbeck T, Hollert H, Brauns A, Keiter S, Schwartz P (2009) Fischpopulationen unter Stress - das Beispiel des Unteren Neckars. Umweltwiss Schadst Forsch 21(2):197-211

Claus E, Neumann-Hensel H, Heininger P, Schwarzbauer J (2009a) Effektorientierte Untersuchungen zur Algentoxizität fraktionierter Porenwässer und Eluate aus Elbesedimenten. Beitragsreihe Wirkungsorientierte Analytik in der Aquatischen Umwelt. Umweltwiss Schadst Forsch 21(3):268-272
Claus E, Heininger P, Schwarzbauer J (2009b) Vorwort. Beitragsreihe Wirkungsorientierte Analytik in der Aquatischen Umwelt. Umweltwiss Schadst Forsch 21(3):238-239

Feiler U, Ahlf W, Hoess S, Hollert H, Neumann-Hensel H, Meller M, Weber J, Heininger P (2005) The SeKT joint research project: definition of reference conditions, control sediments and toxicity thresholds for limnic sediment contact tests. Environ Sci Pollut Res 12:257-258

Feiler U, Claus E, Spira D, Heininger P (2009) Der Einsatz von Pflanzentests bei der Sedimentbewertung. Beitragsreihe Wirkungsorientierte Analytik in der Aquatischen Umwelt. Umweltwiss Schadst Forsch 21(3):264-267

Hollert H, Heise S, Keiter S, Heininger P, Förstner U (2007) Wasserrahmenrichtlinie - Fortschritte und Defizite. Umweltwiss Schadst Forsch 19:58-70

Hollert H, Ernst M, Ahlf W, Dürr M, Erdinger L, Grund S, Keiter S, Kosmehl T, Seiler T-B, Wölz J, Braunbeck T (2009a) Strategien zur Sedimentbewertung - ein Überblick. Umweltwiss Schadst Forsch 21(2):160-176

Hollert H, Keiter S, Böttcher M, Grund S, Seitz N, Otte J, Bluhm J, Wurm K, Hecker M, Higley E, Giesy J, Takner H, van Bavel B, Engwall M, Reifferscheid G, Manz W, Erdinger L, Schulze T, Luebcke-van Varel U, Kammann U, Schöneberger R, Suter M, Brack W, Strähle U, Braunbeck T (2009b) Eine Weight-of-Evidence-Studie zur Bewertung der Sedimentbelastung und des Fischrückgangs in der Oberen Donau. Beitragsreihe Wirkungsorientierte Analytik in der Aquatischen Umwelt. Umweltwiss Schadst Forsch 21(3):260-263

Keiter S, Böttcher M, Grund S, Seitz N, Braunbeck T, Hollert H (2009) Der Fischrückgang in der oberen Donau. Umweltwiss Schadst Forsch 21(2):186-196

Lehn H, Parodi O (2009) Wasser - elementare und strategische Ressource des 21. Jahrhunderts - I. Eine Bestandsaufnahme. Beitragsreihe Wasser \& Nachhaltige Entwicklung. Umweltwiss Schadst Forsch 21(3):273-282

EC (European Commission) (2007) Regulation (EC) No 1907/2006 of the European Parliament and of the Council of 18 December 2006 concerning the Registration, Evaluation, Authorization and Restriction of Chemicals (REACH). Official J Eur Union L 136, v 3-280

Reifferscheid G, Claus E, Manz W (2005) Spezifische toxische Wirkungen in der Sedimentbewertung. Nachweis spezifischer Wirkungen und Identifikation verursachender Stoffe. Wasser J 103:3-32

Reifferscheid G, Buchinger S, Claus E (2009) Das Potential genetisch modifizierter und gentechnisch konstruierter Organismen in der wirkungsbezogenen Analytik. Beitragsreihe Wirkungsorientierte Analytik in der Aquatischen Umwelt. Umweltwiss Schadst Forsch 21(3):256-259

Rüdel H, Steinhanses J, Müller J, Schröter-Kermani C (2009) Retrospektives Monitoring von Organozinnverbindungen in biologischen Proben aus Nord- und Ostsee - sind die Anwendungsbeschränkungen erfolgreich? Umweltwiss Schadst Forsch 21(3):283-292

Schaeffer A, Hollert H, Ratte H-T, Ross Nickoll M, Filser J, Matthies M, Oehlmann J, Scheringer M, Schulz R, Seitz A (2009) An indispensable asset at risk: merits and needs of chemicals-related environmental sciences. Environ Sci Pollut Res. DOI: 10.1007/ s11356-009-0157-x

Schwarzenbach RP, Escher BI, Fenner K, Hofstetter TB, Johnson CA, von Gunten U, Wehrli B (2006) The challenge of micropollutants in aquatic systems. Science 313:1072-1077 